\title{
Desenvolvimento de máquina descascadora de pinhão
}

\author{
Alberto Kazushi Nagaoka ${ }^{1}$, Fernando Cesar Bauer ${ }^{2}$, Guinther Hugo Grudtner ${ }^{3}$, Suelen Silva de Jesus ${ }^{3}$ e Marilda da Penha
}

Teixeira Nagaoka ${ }^{4}$

\begin{abstract}
Resumo - A cadeia produtiva do pinhão envolve as etapas de colheita, descasque, industrialização e comercialização. Uma das dificuldades mais encontradas é a falta de equipamentos capazes de descascar o produto, que poderiam aumentar a capacidade de trabalho na atividade. $O$ descascamento é um processo demorado e difícil, necessitando de mão de obra intensiva. Este trabalho teve como principal objetivo desenvolver uma máquina descascadora de pinhão, com metodologia voltada ao desenvolvimento de produtos industriais, composta por diferentes etapas: planejamento do projeto; projeto informacional; projeto conceitual; projeto preliminar e projeto detalhado. A aplicação da sistemática adotada permitiu efetividade no desenvolvimento do projeto e na construção do protótipo. A máquina descascadora de pinhão utilizando cilindro de ranhuras retas com $3 \mathrm{~cm}$ de diâmetro ofereceu melhor desempenho, com capacidade de manipulação de $24,6 \mathrm{~kg} \mathrm{~h}^{-1}$ e eficácia de $61 \%$. A melhor velocidade obtida foi de $0,0157 \mathrm{~m} \mathrm{~s}^{-1}$, sendo considerada adequada às condições de uso dos produtores familiares da Serra Catarinense.
\end{abstract}

Termos de indexação: Araucaria angustifolia; semente; mecanização.

\section{Development of a "pinhão" (brazilian pine nut) peeling machine}

Abstract - The pine nut production chain involves the harvesting, peeling, industrialization and commercialization. One of the bottlenecks pointed by producers is the lack of an equipment capable of peeling the product, which would increase the work capacity of the activity. The peeling the seed is a difficult and time-consuming process, requiring intense labor use. The main objective of this research is to develop a pine nut peeling machine. It was based on a methodology focused on industrial products' development and was composed by different stages: project planning; informational project; conceptual project; preliminary project; and detailed project. The systematic application adopted has allowed effectiveness in the project's development and in the prototype's construction. The pine nut peeling machine that had $3 \mathrm{~cm}$ diameter straight grooved cylinders offered the best performance having a handling capacity of $24.6 \mathrm{~kg} \mathrm{~h}^{-1}$ and an efficiency of $61 \%$. The best speed obtained was $0,0157 \mathrm{~m} \mathrm{~s}^{-1}$, considered adequate to the conditions of use of the family producers of Serra Catarinense.

Index terms: Araucaria angustifolia; seed; mechanization.

\section{Introdução}

Nota-se no decorrer dos últimos anos um grande incentivo a trabalhos de proteção ambiental e ao uso consciente dos recursos naturais, principalmente daqueles já ameaçados. Neste contexto, destaca-se a cadeia produtiva do pinhão, semente da araucária (Araucaria angustifolia), uma espécie gravemente ameaçada de extinção. Os estudos têm sido realizados de diversas formas, analisando aspectos ecológicos, sociais, mercadológicos, produtivos e de regulamentação. Contudo, apesar dos bons resultados obtidos, ainda faltam informações em algumas etapas do processo, especialmente naquelas relacionadas à tecnologia e desenvolvimento de máquinas adequadas aos procedimentos envolvidos na cadeia produtiva do pinhão.

$\mathrm{Na}$ serra catarinense, a extração, processamento e comercialização da semente são atividades tradicionais importantes, sendo parte da fonte de renda de muitas famílias (SILVEIRA et al., 2011). O pinhão é utilizado principalmente como alimento humano, apresentando alta qualidade nutricional (HENRIQUEZ et al., 2008). A cadeia produtiva do pinhão resume-se em colheita, descascamento e comercialização, sendo os dois primeiros procedimentos demorados e difíceis. Há, ainda, dificuldade resultante da escassez de mão de obra e da falta de equipamentos adequados, especialmente relacionados ao descascamento das sementes. Segundo Nagaoka et al. (2016), muitos produtores rurais da região serrana catarinense realizam a extração do pinhão e cerca de $86 \%$ das famílias entrevistadas consideraram que qualquer tipo de desenvolvimento técnico na cadeia extrativista contribuiria para o avanço da atividade. Com isso, buscamos desenvolver e construir uma máquina descascadora de pinhão adequada às condições das famílias e cooperativas ligadas à atividade extrativista.

${ }^{3}$ Acadêmico do Curso de Agronomia, UFSC, campus Florianópolis, e-mail: guinther_grudtner@hotmail.com; suelen_inuu@hotmail.com.

${ }^{4}$ Economista, Dr., IFSC/ Professora temporária associada ao Centro de Referência em Formação e EaD (CERFEAD), 88015-640 Florianópolis, SC, fone: (48) 98492-7473, e-mail: marildanagaoka@yahoo.com.br. 


\section{Material e métodos}

O projeto foi desenvolvido de 2014 até 2017 no Laboratório de Instrumentação Agrícola do Centro de Ciências Agrárias (CCA) da Universidade Federal de Santa Catarina (UFSC), em parceria com a Cooperativa Ecoserra, de Lages, SC. Sua metodologia foi baseada na proposta por Back et al. (2008), apresentada na Figura 1.

As fases do desenvolvimento do projeto foram:

\section{1 - Planejamento}

Organização e realização de reuniões com os integrantes da pesquisa, visando definir o objetivo geral, a formalização do projeto e a atuação das partes envolvidas.

\section{2 - Projeto informacional}

O objetivo nesta etapa foi obter informações para nortear o início dos trabalhos de desenvolvimento. Foram realizadas 14 visitas técnicas em comunidades distintas dos municípios catarinenses de Urubici e Painel, considerados polos de extração de pinhão. Nessas visitas, foram também realizadas pesquisas exploratórias com os agricultores envolvidos na extração, buscando conhecer suas características de vida e necessidades em relação à mecanização agrícola. Questionários semiestruturados foram aplicados, com o objetivo de avaliar quais os principais atributos necessários no processo de mecanização da cadeia produtiva do pinhão. $O$ questionário foi composto de 23 perguntas, divididas nos segmentos: pessoal; extração do pinhão na propriedade; me-

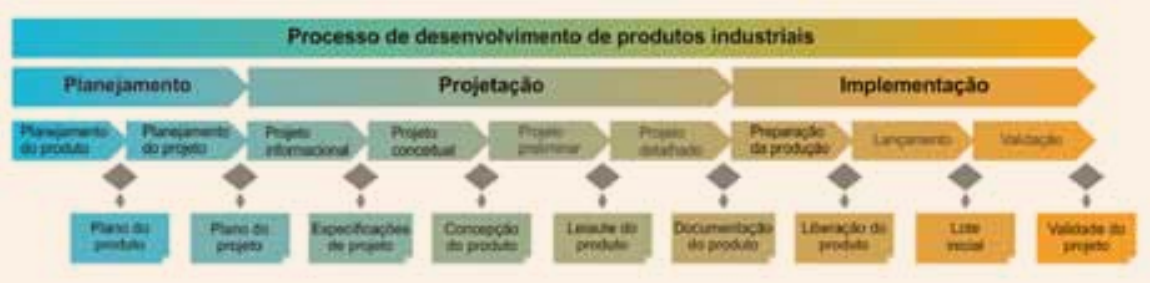

Figura 1 - Resumo do processo de desenvolvimento de produtos industriais. Adaptado de Romano (2003) e Back et al. (2008)

Figure 1 - Summary of the process of development of industrial products. Adapted from Romano (2003) and Back et al. (2008) canização da cadeia produtiva; e necessidade de máquinas para a atividade. Os resultados foram avaliados por meio de gráficos ilustrativos que possibilitaram a sucessão do trabalho.

\section{3 - Projeto conceitual}

O projeto Conceitual teve função de avaliar todas as etapas que a descascadora deveria cumprir e as especificações dos componentes, realizando pesquisa em empresas para auxiliar na construção da máquina. Também fez parte dessa etapa a coleta de documentos para solicitação de depósito de patente de invenção ou modelo de utilidade para a denominação "Máquina Descascadora de Pinhão". Também houve a realização de desenhos de peças e detalhes e estruturas da máquina.

Ainda nesta etapa, realizaram-se testes com a sua maquete, tornando possível obter dados que apontassem melhorias a serem realizadas. Para que pudesse ser realizado o dimensionamento do protótipo e de suas peças constituintes finais, realizou-se uma avaliação das características físicas do pinhão para obter dados sobre o dimensionamento dos cilindros descascadores. As sementes de araucária usadas foram adquiridas em Bom Retiro, Florianópolis e São Joaquim, com o objetivo de obter tamanhos e formatos distintos. Foram utilizadas 50 sementes de cada amostra, avaliando as variáveis peso (g), largura máxima $(\mathrm{mm})$, espessura máxima $(\mathrm{mm})$, comprimento $(\mathrm{mm})$, volume $(\mathrm{mL})$, densidade $\left(\mathrm{g} / \mathrm{cm}^{-3}\right)$ e espessura da coriácea, medida a cada 0,5 mm ao longo da semente. Para avaliação dos dados, aplicou-se o teste de Tukey a $5 \%$ de probabilidade e utilizou-se o Software Assistat 7.7. Para a aferição da variável peso, utilizou-se uma balança digital da marca Exacta, modelo BR-3200AS com precisão de $+/-0,1 \mathrm{~g}$. Para as medições milimétricas, utilizou-se um paquímetro analógico da marca Stareer com precisão de +/- 0,02 mm. O volume foi obtido com o uso de uma proveta de $100 \mathrm{ml}$, na qual a semente foi imersa e o valor aferido a partir da diferença entre o volume inicial e final. A densidade foi mensurada a partir da seguinte equação:

$$
\text { Densidade }=\frac{\text { Massa da Amostra }(\mathrm{g})}{\text { Volume da amostra (ml) }}
$$

\section{4-Projeto preliminar}

A quarta fase objetivou construir, testar e avaliar o desempenho do protótipo da descascadora de pinhão. TEsta etapa compreendeu desde o desenvolvimento do modelo inicial, com a escoIha de materiais de construção, dos processos de manufatura e a definição de dimensões até os ensaios. Para realizar testes de desempenho da descascadora foram utilizados: 2.400 sementes de araucária; protótipo da descascadora de pinhão; duas panelas de pressão; balança de precisão digital; cronômetro; e tacômetro de contato. Inicialmente os pinhões foram pesados e em seguida cozidos por 50 minutos.

Após o cozimento, as sementes foram mantidas aquecidas e foram descascadas com 4 diferentes diâmetros do cilindro descascador $(3,4,5$ e $6 \mathrm{~cm}), 3$ tipos de ranhuras (alternada, helicoidal e reta) e 4 velocidades periféricas distintas $\left(0,0157 \mathrm{~m} \mathrm{~s}^{-1}, 0,0314 \mathrm{~m} \mathrm{~s}^{-1}, 0,0471 \mathrm{~m}\right.$ $\mathrm{s}^{-1} \mathrm{e} 0,0628 \mathrm{~m} \mathrm{~s}^{-1}$ ), totalizando 48 testes. Para cada teste de velocidade, diâmetro e ranhura do cilindro foram usadas 50 sementes, colocando-as de duas em duas na entrada do protótipo da máquina. Os dados avaliados foram: tempo de descasque (s); os pesos antes e depois de cozer, do pinhão descascado, da casca, da amostra descascada inteira e da amostra descascada quebrada (g); número de pinhões descascados inteiros e de pinhões descascados quebrados. A partir dos dados obtidos, calculou-se para cada teste: eficácia (\%), capacidade de manipulação efetiva $\left(\mathrm{kg} \mathrm{h}^{-1}\right)$, capacidade de produção efetiva $\left(\mathrm{kg} \mathrm{h}^{-1}\right)$, quebra (\%) e velocidade linear, usando, respectivamente, as seguintes equações: 


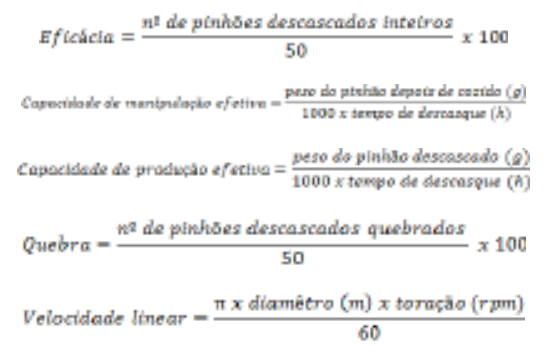

Os dados obtidos foram analisados considerando o delineamento experimental em blocos, segundo parcelas subdivididas. Em cada parcela havia o diâmetro dos cilindros $(\mathrm{d} 1=3 \mathrm{~cm}$, $\mathrm{d} 2=4 \mathrm{~cm}, \mathrm{~d} 3=5 \mathrm{~cm}$ e $\mathrm{d} 4=6 \mathrm{~cm})$, na primeira subdivisão, o desenho das ranhuras ( $\mathrm{r} 1=$ alternada, $\mathrm{r} 2=$ helicoidal e r3 = reta) e, na subdivisão desta, as velocidades $\left(\mathrm{v} 1=0,0157 \mathrm{~ms}^{-1}\right.$, $\mathrm{v} 2=0,0314 \mathrm{~ms}^{-1}, \mathrm{v} 3=0,0471 \mathrm{~ms}^{-1} \mathrm{e}$ $\left.\mathrm{v} 4=0,0628 \mathrm{~ms}^{-1}\right)$; com 50 repetições.

\section{5 - Projeto detalhado}

A partir dos resultados obtidos na fase anterior, a máquina descascadora final foi construída. Após a aprovação do pedido de modelo de utilidade, o produto foi encaminhado para a Cooperativa Ecoserra para validação.

\section{Resultado e discussão}

A Figura 2 indica que, dentre as propostas de desenvolvimento de máquinas para a atividade, $50 \%$ dos entrevistados deram prioridade para aquisição de uma colhedora de pinhão, devido aos relatos de acidentes ocorridos todos os anos nesta operação, que exige habilidade para subir nas árvores. A segunda prioridade mais escolhida foi a descascadora, com $14 \%$. Os demais (36\%), mostraram-se adeptos ao desenvolvimento de qualquer máquina voltada a área. Baseado na movimentação de volumes consideráveis de pinhão processado pela Cooperativa e na fixação do objetivo do trabalho no desenvolvimento de máquina descascadora, deuse preferência à segunda prioridade.

Outro fato que alavancou o projeto foi a verificação de a colheita e o descascamento serem as etapas que mais influenciam o custo total de produção do pinhão, principalmente pela falta de equipamento adequado. Observou-se

\section{Ordem de importância na aquisição de máquinas}

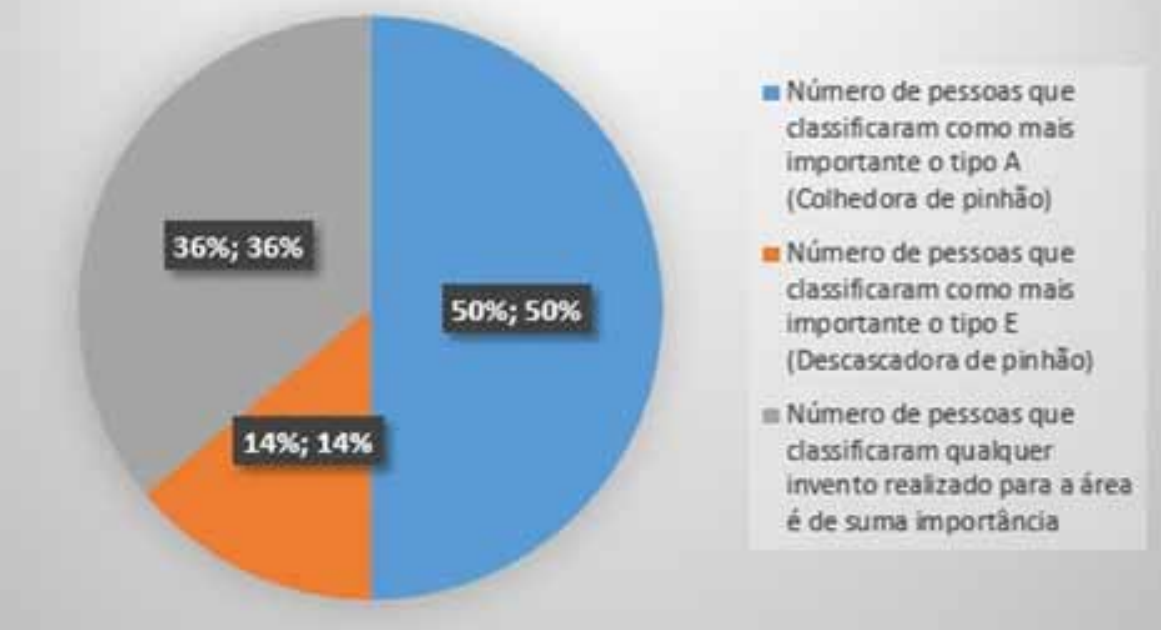

Figura 2 - Classificação das prioridades de propostas de máquinas voltadas a cadeia produtiva do pinhão na Serra Catarinense

Figure 2 - Classification of the priorities of proposals of machines directed to the productive chain of pinion in Serra Catarinense

que, por ser lenta, a operação de descascamento representa um gargalo no processo de industrialização do pinhão. Notou-se ainda que os agricultores que consideraram necessário adquirir uma máquina descascadora foram influenciados diretamente pelo custo, pois o extrativismo é restrito a uma época do ano, e o seu processamento geralmente é feito de forma não escalonada. Cerca de $43 \%$ dos entrevistados apresentaram interesse em adquirir a máquina descascadora caso seu preço fosse igual ou inferior a $R \$ 2.500,00$.

$\mathrm{Na}$ terceira etapa, foram realizados testes em uma maquete, na qual houve adaptação de um cilindro laminador de massa acoplado a um motor elétrico para realizar o descascamento do pinhão, cujos resultados estão na Figura 3. Verifica-se que o desempenho deste primeiro modelo de máquina demonstrou potencial significativo, apresentando capacidade de produção efetiva de $3,33 \mathrm{~kg} \mathrm{~h}^{-1}$ e de manipulação efetiva de $6,44 \mathrm{kgh}^{-1}$, valores superiores aos encontrados por Nagaoka et al. (2014), que avaliou um protótipo similar. Nos seus resultados, a capacidade de produção encontrada foi de $2,1 \mathrm{k} \mathrm{gh}^{-1}$ e a capacidade de manipulação efetiva, de $4,3 \mathrm{k} \mathrm{gh}^{-1}$. Porém, notou-se que os resultados encontrados no atual trabalho po- deriam ser melhores caso fosse projetada uma máquina mais eficaz, realizando estudos mais aprofundados sobre as características da semente. Outro fato que chamou atenção foi o baixo consumo de energia, de $0,14 \mathrm{~kW} / \mathrm{h}$, indicando um baixo gasto energético no processo de descasque, próximo a $R \$ 0,048 \mathrm{~h}^{-1}$.

Tendo em vista a busca por uma máquina com maior capacidade de produção, foram avaliadas as características físicas do pinhão, para dimensionar os cilindros descascadores. A Tabela 1 mostra que apenas as amostras de Bom Retiro apresentaram diferença estatística para as variáveis peso, largura máxima e volume. As amostras de Florianópolis apresentaram diferença no comprimento. Henríquez et al. (2008), estudando as características físicas do pinhão, concluiu que a semente mede de 3,5 a $4,5 \mathrm{~cm}$ de comprimento e de 1,2 a $2 \mathrm{~cm}$ de diâmetro, tendo peso médio de 3,5 a 3,8g, valores diferentes dos encontrados neste trabalho. Isso reforça a existência de variações consideráveis nas características de sementes obtidas em regiões distintas.

Dessa forma, a análise indicou que existem diferenças físicas nas sementes de origens diversas, revelando que a máquina precisaria de regulagens no distanciamento dos cilindros para poder 


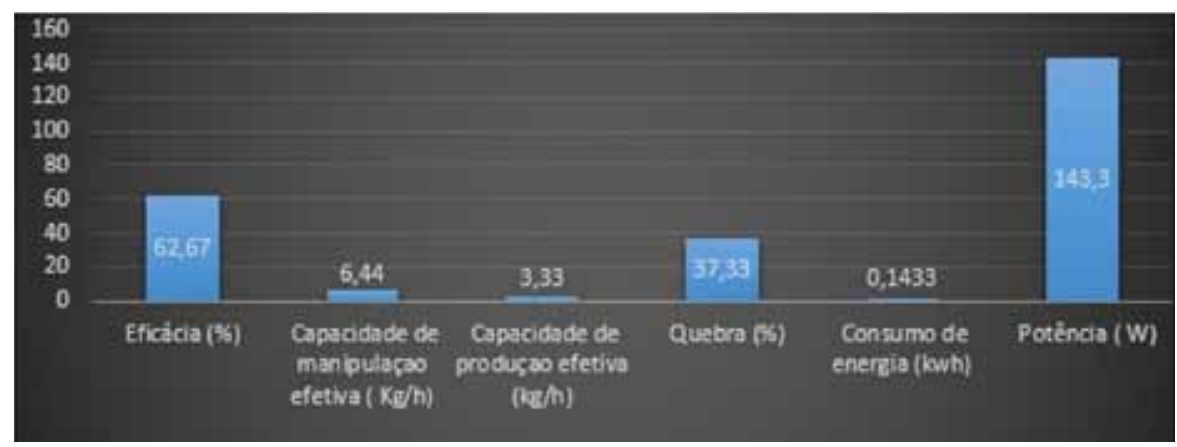

Figura 3 - Resultados médios do ensaio com a maquete da máquina adaptada para descascamento de pinhão

Figure 3 - Average results of the test with the model of the machine adapted for peeling of pinion

Tabela 1 - Média das variáveis peso, largura máxima, espessura máxima, comprimento, volume e densidade para as três origens

Table 1 - Mean of the variables weight, maximum width, maximum thickness, length, volume and density for the three or

\begin{tabular}{lcccccc}
\hline Origem & Peso $(\mathbf{g})$ & $\begin{array}{c}\text { Largura } \\
\text { máxima } \\
(\mathbf{m m})\end{array}$ & $\begin{array}{c}\text { Espessura } \\
\text { máxima } \\
(\mathbf{m m})\end{array}$ & $\begin{array}{c}\text { Comprimento } \\
(\mathbf{m m})\end{array}$ & $\begin{array}{c}\text { Volume } \\
(\mathbf{m l})\end{array}$ & $\begin{array}{c}\text { Densidade } \\
\left(\mathbf{g} / \mathbf{c m}^{3}\right)\end{array}$ \\
\hline Bom Retiro & $6,77 \mathrm{a}$ & $20,09 \mathrm{a}$ & $16,12 \mathrm{a}$ & $54,50 \mathrm{a}$ & $6,98 \mathrm{a}$ & $0,96 \mathrm{a}$ \\
São Joaquim & $5,64 \mathrm{~b} \mathrm{~b}$ & $18,93 \mathrm{~b}$ & $16,26 \mathrm{a}$ & $54,22 \mathrm{a}$ & $5,76 \mathrm{~b}$ & $0,98 \mathrm{a}$ \\
Florianópolis & $5,43 \mathrm{~b} \mathrm{~b}$ & $20,03 \mathrm{ab}$ & $15,75 \mathrm{a}$ & $50,41 \mathrm{~b}$ & $5,83 \mathrm{~b}$ & $0,92 \mathrm{a}$ \\
\hline CV (\%) & 25,38 & 12,17 & 13,51 & 10,31 & 25,04 & 13,51 \\
\hline
\end{tabular}

(1) Médias seguidas pela mesma letra nas colunas não diferem estatisticamente entre si pelo teste de

Tukey a $5 \%$ de probabilidade.

(2) CV (\%)= Coeficiente de variação. onde há a tração inicial dos cilindros para o descasque, foi o melhor. A partir da análise das características físicas do pinhão, dimensionaram-se os cilindros descascadores (figuras $5 \mathrm{a}$ e $5 \mathrm{~b}$ ) com diferentes diâmetros e foi projetado o protótipo de máquina descascadora de pinhão baseado no modelo da maquete.

A Figura 6a representa o protótipo da descascadora de pinhão, desenhado com auxílio do software SolidWorks. A Figura $6 \mathrm{~b}$ mostra o protótipo construído após orçamento em diferentes empresas de metalurgia.

Depois de construir o protótipo e os diferentes cilindros, foram realizados os testes, combinando os quatro diâmetros com diferentes ranhuras e velocidades. Os resultados estão descritos na Tabela 4. Para o cilindro $1(3 \mathrm{~cm})$, a média geral da capacidade de produção efetiva foi de $7,24 \mathrm{~kg} \mathrm{~h}^{-1}$, com eficácia de $42,83 \%$. A média geral dos resultados para o cilindro $2(4 \mathrm{~cm})$, com as diferentes ranhuras e velocidades, foi de $6,23 \mathrm{~kg} \mathrm{~h}^{-1}$ e $43,66 \%$, respectivamente. Para o ci- realizar o descasque de forma homogênea. As tabelas 2 e 3 apresentam análises estatísticas da espessura da coriácea de 0,0 até $3,0 \mathrm{~cm}$ e de 3,5 até $6,0 \mathrm{~cm}$ ao longo da semente, respectivamente. As medidas longitudinais aferidas em 1,5 ; 3,$0 ; 3,5 ; 4,0 ; 4,5 ; 5,0$ e $5,5 \mathrm{~cm}$ ao longo da semente apresentaram variação estatística nas diferentes origens. Verificase também que o comprimento de $2 \mathrm{~cm}$ possui os maiores valores de espessura de coriácea.

A partir da obtenção dos valores médios das sementes das três regiões, projetou-se um desenho do formato do pinhão em uma folha milimetrada (Figura 4). Desta forma, pode-se relacionar o diâmetro do cilindro descascador com o formato da semente, possibilitando a visualização e relação da área de contato entre o órgão ativo da máquina e a semente.

Observa-se que os diâmetros que apresentaram relação mais expressiva na área de contato foram os de 3, 4, 5 e $6 \mathrm{~cm}$, com destaque para o de $3 \mathrm{~cm}$, cujo encaixe na parte inicial da coriácea,
Tabela 2 - Média dos valores de espessura da coriácea do pinhão, mensurados do ponto 0 $\mathrm{cm}$ até 3,0 cm ao longo do comprimento da semente

Table 2 - Mean values of thickness of the coriacea of the pinion, measured from $0 \mathrm{~cm}$ to 3.0 $\mathrm{cm}$ along the length of the seed

\begin{tabular}{|c|c|c|c|c|c|c|c|c|}
\hline & \multirow{2}{*}{ Espessura } & \multicolumn{7}{|c|}{ Comprimento $(\mathrm{cm})$} \\
\hline & & 0 & 0,5 & 1 & 1,5 & 2 & 2,5 & 3 \\
\hline \multirow[t]{3}{*}{ Origem } & Bom Retiro (mm) & $6,8 \mathrm{a}$ & $6,8 \mathrm{a}$ & 10,5 a & $14,3 \mathrm{~b}$ & $15,6 a$ & $15,5 \mathrm{a}$ & $14,5 \mathrm{a}$ \\
\hline & São Joaquim (mm) & 7,4 a & $6,8 a$ & $11,8 \mathrm{a}$ & $15,8 \mathrm{a}$ & $15,8 \mathrm{a}$ & $14,8 \mathrm{a}$ & $12,5 \mathrm{~b}$ \\
\hline & Florianópolis (mm) & 7,2 a & $7,3 \mathrm{a}$ & $11,3 \mathrm{a}$ & $15,1 a b$ & $15,3 \mathrm{a}$ & $14,4 \mathrm{a}$ & $12,9 \mathrm{~b}$ \\
\hline & CV (\%) & 21.9 & 23.93 & 25.12 & 14.47 & 14.59 & 16.36 & 20.06 \\
\hline
\end{tabular}

(1) Médias seguidas pela mesma letra nas colunas não diferem estatisticamente entre si pelo teste de Tukey a $5 \%$ de probabilidade.

(2) $\mathrm{CV}(\%)=$ Coeficiente de variação.

Tabela 3 - Média dos valores de espessura da coriácea do pinhão, mensurados do ponto $3,5 \mathrm{~cm}$ até $6,0 \mathrm{~cm}$ ao longo do comprimento da semente

Table 3 - Mean values of thickness of the coriacea of the pinion, measured from $3.5 \mathrm{~cm}$ to $6.0 \mathrm{~cm}$ along the length of the seed

\begin{tabular}{cccccccc}
\hline & & \multicolumn{6}{c}{ Comprimento (cm) } \\
\cline { 2 - 8 } Ospessura & $\mathbf{3 , 5}$ & $\mathbf{4}$ & $\mathbf{4 , 5}$ & $\mathbf{5}$ & $\mathbf{5 , 5}$ & $\mathbf{6}$ \\
\cline { 2 - 8 } Origem & Bom Retiro (mm) & 12,98 a & 8,17 a & 5,86 a & 2,88 a & 1,26 a & 0,24 a \\
\cline { 2 - 8 } & São Joaquim (mm) & 9,91 b & 4,30 b & 1,96 b & 0,75 b & 0,23 b & 0,07 a \\
& Florianópolis (mm) & 10,99 b & 5,62 b & 2,85 b & 1,02 b & 0,15 b & 0,00 a \\
\hline & C.V (\%) & 27,28 & 59,00 & 90,73 & 153,00 & 276,10 & 547,71 \\
\hline
\end{tabular}

(1) Médias seguidas pela mesma letra nas colunas não diferem estatisticamente entre si pelo teste de Tukey a $5 \%$ de probabilidade.

${ }^{(2)} \mathrm{CV}(\%)=$ Coeficiente de variação. 


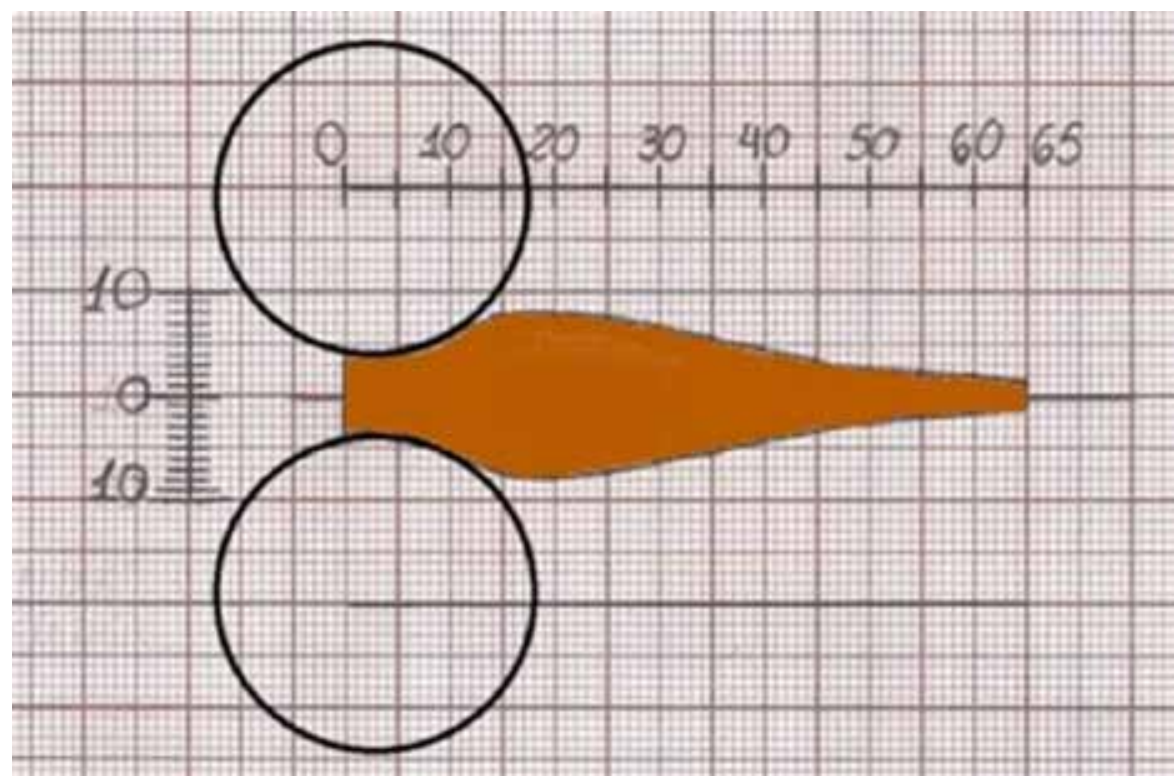

Figura 4 - Projeção em perfil da semente do pinhão ajustado ao cilindro de descasque com diâmetro de 3 centímetros (Escala 1:1)

Figure 4 - Profile projection of the pinion seed adjusted to the husking cylinder with a diameter of 3 centimeters (1: 1 scale) lindro $3(5 \mathrm{~cm})$, com diferentes ranhuras e velocidades o resultado médio da capacidade de produção efetiva foi de $9,07 \mathrm{~kg} / \mathrm{h}^{-1}$, com eficácia de $49,83 \%$. Os últimos testes, realizados com o cilindro $4(6 \mathrm{~cm})$ tiveram valor médio de capacidade de produção efetiva de $7,81 \mathrm{~kg} / \mathrm{h}^{-1}$ e eficácia de $29 \%$.

Tendo em vista que, para a atividade do descascamento do pinhão, é essencial haver maior eficácia e capacidade de produção, além de manipulação efetiva com poucas quebras, o diâmetro 1 (cm) com ranhura 3 (reta) obteve meIhor desempenho. Ele apresentou bons resultados em relação a capacidade de manipulação $\left(24,61 \mathrm{~kg} / \mathrm{h}^{-1}\right)$ e produção efetiva $\left(12,12 \mathrm{~kg} / \mathrm{h}^{-1}\right)$ além de boa eficácia (61\%). Para os dados obtidos através da avaliação das diferentes velocidades,
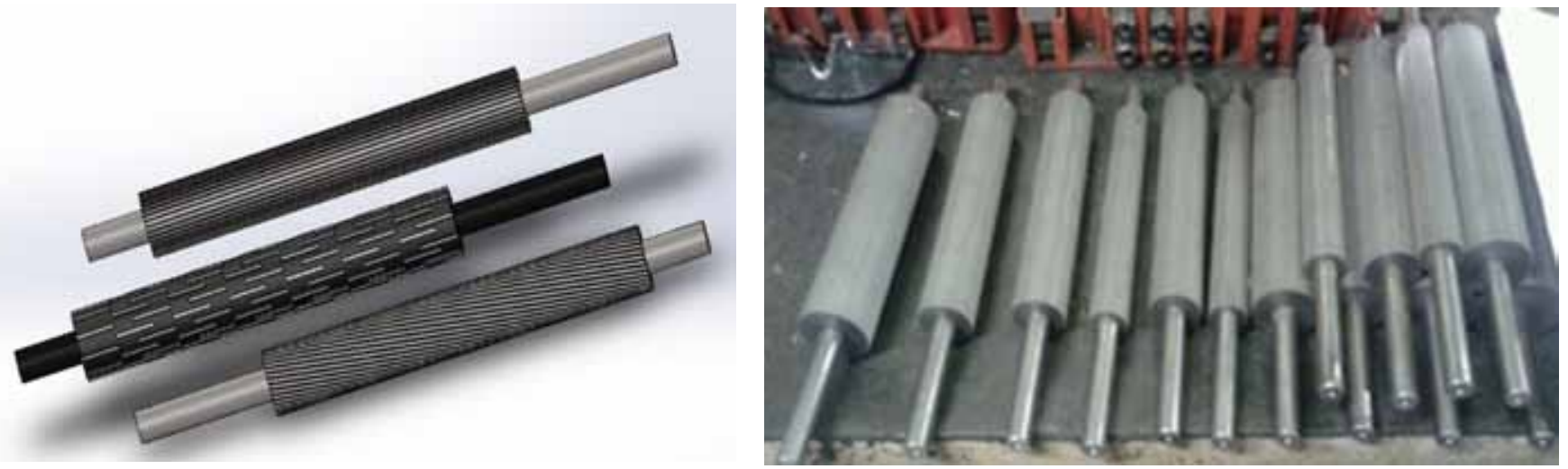

Figura 5 - Desenho dos cilindros de descasque com três ranhuras diferentes (a) e cilindros construídos com quatro diâmetros e três ranhuras de superfície diferentes (b)

Figure 5 - Design of the debarking cylinders with three different grooves (a) and cylinders constructed with four diameters and three different surface grooves (b)

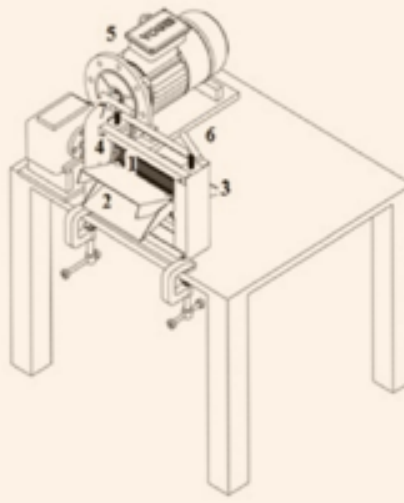

1- Cilindro superior

2- Moega de alimentação

3- Moega de saida de cascas

4- Engrenagens de acionamento

5- Motor elétrico

6- Base da máquina

7- Regulagem de pressão

Figura 6 - Desenho do protótipo de descascadora de pinhão (a) e protótipo da descascadora de pinhão (b)

Figure 6 - Design of the prototype of the pinion peeler (a) and prototype of the pinion peeler (b) 
nota-se que a velocidade correspondente a $0,0157 \mathrm{~m} \mathrm{~s}^{-1}$, combinada com o diâmetro 1 e a ranhura reta, apresentou a melhor eficácia (88\%). Sua capacidade de produção efetiva foi de $12,74 \mathrm{~kg} \mathrm{~h}^{-1} \mathrm{e}$ a de manipulação específica foi $21,7 \mathrm{~kg}$ $\mathrm{h}^{-1}$, valores aproximadamente quatro vezes maiores que os encontrados na avaliação do primeiro modelo da máquina.

\section{Conclusão}

A metodologia adotada possibilitou conhecer a cadeia produtiva do pinhão, as operações envolvidas após a colheita e principalmente os problemas relacionados à industrialização da produção. A máquina descascadora de pinhão desenvolvida neste projeto superou o desempenho operacional manual dos produtores da região da Serra Catarinense, servindo de alternativa viável para o processamento do pinhão. Torna-se, portanto, forma de resolução de problemas ligados à industrialização.

\section{Agradecimentos}

Agradecemos o Instituto Oi Futuro

Tabela 4 - Síntese dos valores de capacidade de produção efetiva (CPE), capacidade de manipulação efetiva (CME), eficácia e quebra para as variáveis diâmetro (D), ranhura e velocidade (V)

Table 4 - Synthesis of effective production capacity (CPE), effective handling capacity (CHE), efficacy and breakage for the variables diameter (D), slot and velocity (V)

\begin{tabular}{|c|c|c|c|c|c|}
\hline $\mathrm{D}(\mathrm{cm})$ & Ranhura & CPE $\left(\mathrm{kg} / \mathrm{h}^{-1}\right)$ & CME $\left(\mathrm{kg} / \mathrm{h}^{-1}\right)$ & Eficácia (\%) & Quebra (\%) \\
\hline 3 & alternada & 5,40 & 11,15 & 37,5 & 62,5 \\
\hline 3 & helicoidal & 4,20 & 10,37 & 30 & 70 \\
\hline 3 & reta & 12,12 & 24,61 & 61 & 39 \\
\hline Média & & 7,24 & 15,38 & 42,83 & 57,16 \\
\hline 4 & alternada & 5,78 & 11,73 & 46,5 & 53,5 \\
\hline 4 & helicoidal & 4,25 & 9,96 & 29,5 & 70,5 \\
\hline 4 & reta & 8,67 & 16,50 & 55 & 45 \\
\hline Média & & 6,23 & 12,73 & 43,66 & 56,33 \\
\hline 5 & alternada & 4,24 & 28,05 & 53,5 & 46,5 \\
\hline 5 & helicoidal & 4,14 & 13,22 & 33 & 67 \\
\hline 5 & reta & 8,84 & 16,13 & 63 & 37 \\
\hline Média & & 5,74 & 19,17 & 49,83 & 50,16 \\
\hline 6 & alternada & 11,13 & 23,56 & 26,5 & 48,5 \\
\hline 6 & helicoidal & 5,54 & 14,28 & 22 & 78 \\
\hline 6 & reta & 6,77 & 15,05 & 38,5 & 61,5 \\
\hline Média & & 7,81 & 17,60 & 29,00 & 62,66 \\
\hline$V\left(\mathrm{~m} / \mathrm{s}^{-1}\right)$ & Ranhura & CPE $\left(\mathrm{kg} / \mathrm{h}^{-1}\right)$ & CME $\left(\mathrm{kg} / \mathrm{h}^{-1}\right)$ & Eficácia (\%) & Quebra (\%) \\
\hline 0,0157 & reta & 12,419 & 21,741 & 88 & 12 \\
\hline 0,0314 & reta & 12,229 & 23,718 & 62 & 38 \\
\hline 0,0471 & reta & 14,698 & 30,895 & 50 & 50 \\
\hline 0,0628 & reta & 9,136 & 22,088 & 44 & 56 \\
\hline
\end{tabular}

e a Cooperativa Ecoserra, e o Departamento de Engenharia Rural do CCA/ UFSC, que possibilitaram a realização deste trabalho.

\section{Referências bibliográficas}

BACK, N.; OGLIARI, A.; DIAS, A.; SILVA, J.C. Projeto integrado de produtos: planejamento, concepção e modelagem. Barueri: Manole, 2008. 595 p.

HENRÍQUEZ, C.; ESCOBAR, B.; FIGUEROLLA, F.; CHIFFELLE, I.; SPEISKY, H.; ESTEVEZ, A.M. "Characterization of piñon seed (Araucaria araucana (Mol) K. Koch) and the isolated starch from the seed". Food Chemistry, Barking, v. 107, n. 2, p. 592-601, fev. 2008. Disponível em: <https://pubag.nal. usda.gov/catalog/716256>. Acesso em: 2 ago. 2017.

NAGAOKA, A.K.; BAUER, F.C.; FERREIRA, L.B.; BOOM, G.L.; MOREIRA, T.E. Ensaio de um protótipo de máquina descascadora de pinhão. In: Congresso regional de iniciação científica e tecnológica em engenharia, 26, 2014, Alegrete. Anais... Alegrete: Cricte, 2014.

NAGAOKA, A. K.; BAUER, F.C.; GRUDTNER, G.H.; JESUS, S.S.; NAGAOKA, M.P.T. Identificação de atributos que influenciam na mecanização do pinhão da região serrana de Santa Catarina. In: Congresso brasileiro de engenharia agrícola, 44, 2016, Florianópolis. Anais... Florianópolis: Sbea, 2016. Disponível em: <https://bit.ly/2wqFTkM>. Aceso em: 9 ago. 2017.

ROMANO, L. N. Modelo de referência para o processo de desenvolvimento de máquinas agrícolas. 2003. 321 p. Tese (Doutorado em Engenharia Mecânica)-Centro Tecnológico, Universidade Federal de Santa Catarina, Florianópolis, 2003.

SILVEIRA, E.R.; JAMHOUR, J.; FERONATO, M.L.; XAVIER, E.; CALGARO, E.V.S. Situação das famílias na extração e comercialização do pinhão no sudoeste do Paraná. Synergismus Scyentifica, UTFPR, Pato Branco, v. 6, n. 1, jan. 2011. Disponível em $:<$ https://bit. ly/2BQJzCr>. Acesso em: 3 ago. 2017. 